\title{
Técnicas de coleta de besouros copronecrófagos no Cerrado ${ }^{(1)}$
}

\author{
Michelle Silva Milhomem ${ }^{(2)}$, Fernando Zagury Vaz de Mello $^{(3)}$ e Ivone Rezende Diniz ${ }^{(2)}$
}

Resumo - Métodos de coleta passiva, com o uso de armadilhas, têm sido utilizados na coleta de besouros da família Scarabaeidae. No Cerrado existem poucos estudos sobre estes insetos, apesar da sua importância para o ecossistema e para o controle biológico de pragas do gado bovino. O objetivo deste trabalho foi avaliar técnicas de coleta de besouros copronecrófagos (Coleoptera: Scarabaeidae sensu stricto) com armadilha de interceptação de vôo e armadilha de queda com fezes e com carcaça. O experimento foi realizado em três fitofisionomias (campo sujo, cerrado sensu stricto e mata de galeria) da Reserva Ecológica do IBGE (Instituto Brasileiro de Geografia e Estatística), a $35 \mathrm{~km}$ de Brasília, DF, na época das chuvas (outubro/1999 a janeiro/2000) e da seca (maio a agosto/2000). A armadilha de queda com isca de fezes e o campo sujo apresentaram maior riqueza e abundância de espécies. Houve uma associação positiva entre as chuvas e a distribuição temporal dos besouros. A armadilha de queda com isca de fezes humanas é a mais indicada para coletas de besouros copronecrófagos.

Termos para indexação: Coleoptera, Scarabaeidae, savana, armadilha, sazonalidade, controle de pragas.

\section{Collection techniques of dung beetle in the Cerrado, Brazil}

\begin{abstract}
Passive collection methods, with the use of traps, have been used to collect beetles of the Scarabaeidae family. However, in the Cerrado (Brazilian savanna-like vegetation) there are few studies about these insects, despite its importance to the ecosystem and to the biological control of cattle pests. The aim of this work was to evaluate techniques of dung beetle (Coleoptera: Scarabaeidae sensu stricto) collections with the use of flight interception traps, pitfall traps baited with human faeces, and with carcass. The experiment was accomplished in three different phytophysiognomies (low tree and shrub savanna, semi-open scrub mixed with trees and gallery forests) of the Ecological Reserve of IBGE (Instituto Brasileiro de Geografia e Estatística), $35 \mathrm{~km}$ away from Brasília, DF, in the rainy season (October/1999 to January/2000) and in the dry season (May to August/2000). The pitfall trap baited with faeces and the low tree and shrub savanna presented the greatest richness and abundance of species. There was a positive association between rains and the temporal distribution of the beetles. The pitfall trap baited with human faeces is recommended to collect dung beetles.
\end{abstract}

Index terms: Coleoptera, Scarabaeidae, savannas, traps, seasonality, pest control.

(1)Aceito para publicação em 31 de julho de 2003.

Extraído da tese de doutorado do primeiro autor apresentada à Universidade de Brasília, Brasília, DF. Trabalho financiado pelo CNPq.

(2)Universidade de Brasília, Dep. de Zoologia, Campus Universitário Darcy Ribeiro, Asa Norte, CEP 70910900 Brasília, DF. E-mail: ninami@yahoo.com, irdiniz@unb.br

${ }^{(3)}$ Universidade de Lavras, Dep. de Biologia, Campus Universitário, Centro, CEP 37200-000 Lavras, MG. E-mail: scarab@ufla.br

\section{Introdução}

Os besouros que pertencem à família Scarabaeidae sensu stricto ocorrem no Brasil em sete tribos: Ateuchini, Canthonini, Coprini, Eurysternini, Gromphini, Onthophagini e Phanaeini (Zunino, 1985; Hanski \& Cambefort, 1991; Montreuil, 1998). Possuem coloração bastante variável, com tamanho de $2 \mathrm{~mm}$ a $5 \mathrm{~cm}$ e são encontrados em maior abundância durante a estação chuvosa (Halffter \& Matthews, 1966; Hanski 
\& Cambefort, 1991; Lopes et al., 1994).

Os Scarabaeidae s. str. são detritívoros, isto é, promovem a remoção e reentrada de matéria orgânica morta no ciclo de nutrientes, aumentam a aeração do solo prolongando sua capacidade produtiva, ajudam na dispersão de sementes, além de serem utéis na entomologia forense e no controle biológico (Halffter \& Matthews, 1966; Waterhouse, 1974; Flechtmann et al., 1995; Sheperd \& Chapman, 1998). Os Scarabaeidae s. str. são utilizados no controle de pragas bovinas, como os nematóides gastrointestinais e a mosca-dos-chifres (Haematobia irritans (Linnaeus, 1758)), já que essas pragas têm seus ciclos de vida dependentes dos excrementos frescos dos bovinos (Waterhouse, 1974; Flechtmann et al., 1995).

O Cerrado é uma das 25 áreas do mundo consideradas críticas para a conservação, em virtude de sua riqueza biológica e da alta pressão antrópica a que vem sendo submetido (Mittermeier et al., 2000). Apesar disso, estudos sobre a fauna de Coleoptera nos cerrados brasileiros ainda são muito escassos e pontuais (Hertel \& Colli, 1998; Pinheiro et al., 2002).

O método de coleta afeta a quantificação da comunidade e deve revelar as espécies representativas e a abundância relativa de cada uma delas. Deve ser diferente para grupo de insetos pois estes apresentam variabilidade de hábitos e a variabilidade de hábitos das espécies e da vegetação da área de coleta são determinantes na escolha da técnica de coleta para cada grupo de insetos (Campos et al., 2000).

$\mathrm{Na}$ coleta de espécimes de Scarabaeidae s. str. têm sido utilizados métodos de coleta passiva, ou seja, aqueles realizados com o auxílio de uma armadilha com atrativos biológicos ou físicos. Segundo Favila \& Halffter (1997), o método apropriado para monitorar a abundância de insetos desta família consiste de armadilhas de queda iscadas com excrementos, carcaças ou frutos em decomposição, as quais são enterradas no solo. Na coleta de besouros coprófagos, são utilizadas como iscas fezes humanas ou de herbívoros (gado bovino ou cavalo) e, na coleta de besouros necrófagos, um pedaço de peixe ou lula. No Brasil, armadilhas de queda com iscas de fezes humanas ou bovinas e de carcaças (carne bovina em decomposição) e armadilhas luminosas (especificamente a armadilha "Luiz de Queiroz") estão entre as mais usadas em estudos sobre os besouros rola-bosta (Lopes et al., 1994; Flechtmann et al., 1995; Louzada et al., 1996; Louzada \& Lopes, 1997).

Existem poucos estudos na região tropical sobre eficiência de armadilhas e sistemas para coletas de besouros da família Scarabaeidae s. str. ou para detectar possíveis efeitos de remoção em populações locais (Halffter, 1991; Favila \& Halffter, 1997).

O objetivo deste trabalho foi comparar coletas de besouros copronecrófagos da família Scarabaeidae s. str. com armadilha de interceptação de vôo do tipo "janela" e armadilhas de queda com isca de fezes humanas e de carcaça bovina, em três fitofisionomias do Cerrado.

\section{Material e Métodos}

O trabalho foi realizado na Reserva Ecológica do IBGE (Instituto Brasileiro de Geografia e Estatística) (RECORIBGE) $\left(15^{\circ} 55^{\prime} \mathrm{S}\right.$ e $\left.47^{\circ} 51^{\prime} \mathrm{W}\right)$ localizada a $35 \mathrm{~km}$ SE da cidade de Brasília, com 1.300 ha aproximadamente. Foram selecionadas áreas de campo sujo, cerrado sensu stricto e mata de galeria do córrego Monjolo, próximas à divisão leste da reserva com o Jardim Botânico. As distâncias entre as áreas foram de $1.850 \mathrm{~m}$ entre campo sujo e cerrado, $400 \mathrm{~m}$ entre cerrado e mata e $2.000 \mathrm{~m}$ entre mata e campo sujo.

Na coleta de Scarabaeidae s. str. foram utilizadas as armadilhas de interceptação de vôo do tipo "janela" e armadilhas de queda (conhecida também por alçapão ou "pitfall") com iscas de fezes humanas e de carcaça (fígado bovino em decomposição). A "janela" consistiu de um vidro em uma moldura de alumínio $(80 \times 80 \mathrm{~cm})$ afixada longitudinalmente na porção média de uma calha $(80 \times 30 \times 10 \mathrm{~cm})$ com água detergente e formol (3 L). A armadilha de queda era formada por um recipiente de plástico com $12 \mathrm{~cm}$ de diâmetro e $9 \mathrm{~cm}$ de altura, com $300 \mathrm{~mL}$ de água, detergente e formol, enterrado até o nível do solo. As fezes $(15 \mathrm{~g})$ ficaram em outro recipiente de menor tamanho $(5 \mathrm{~cm}$ de diâmetro e $4 \mathrm{~cm}$ de altura) sustentado por um arame fino em dois pontos opostos e fixado no alto do recipiente maior. No caso da isca de carcaça $(15 \mathrm{~g})$, foi utilizado um vasilhame mais resistente $(4 \mathrm{~cm}$ de diâmetro e $6 \mathrm{~cm}$ de altura) sustentado por dois arames finos colocados em cruz no alto do recipiente maior. Foi colocada uma proteção contra a chuva acima da armadilha, formada por um prato de plástico sustentado por arames. 
O estudo foi realizado durante quatro meses na estação chuvosa (outubro, novembro e dezembro de 1999 e janeiro de 2000) e quatro meses na seca (maio a agosto de 2000). No campo sujo e cerrados. str., armadilhas foram mantidas durante dez dias de cada mês (20 a 29), em áreas de $100 \times 100 \mathrm{~m}$, com pontos marcados de 20 em $20 \mathrm{~m}$, totalizando 36 pontos diferentes de coleta. Na mata de galeria, 36 pontos diferentes de coleta foram marcados ao longo de três trilhas, distantes $50 \mathrm{~m}$ entre si, da seguinte maneira: cada trilha de $120 \mathrm{~m}$ tinha 12 pontos de coleta, 6 pontos a $10 \mathrm{~m}$ do lado direito da trilha e 6 pontos a $10 \mathrm{~m}$ do lado esquerdo, com $20 \mathrm{~m}$ entre cada par de pontos. Em cada área foram instaladas 12 armadilhas, dez de queda e duas "janelas", em sete pontos sorteados a cada mês, sendo as "janelas" em dois pontos e as de queda em cinco pontos (cada um com duas armadilhas de queda com iscas diferentes, distantes $2 \mathrm{~m}$ entre si).

A normalidade dos dados foi verificada pelo teste de D’Agostino-Pearson - $\mathrm{K}^{2}$. As diferenças das médias, de cada mês, de abundância (indivíduos/armadilha/dia de coleta) e de riqueza (espécies/armadilha/dia de coleta) entre as armadilhas em cada área, foram avaliadas pelo teste de Kruskal-Wallis - H; as diversidades e equitabilidades das áreas de coleta foram analisadas pelo índice de Shannon H', e as diferenças entre elas, pelo teste t modificado por Hutcheson; e as abundâncias e riquezas foram comparadas entre as áreas e armadilhas pelo teste Qui-quadrado $\left(\chi^{2}\right)$ (Zar, 1999). Os dados de similaridade faunística foram transformados em $\mathrm{Tn}_{\mathrm{ij}}=\left(\ln \left(\mathrm{n}_{\mathrm{ij}}+1\right)\right)$ e analisados pelo índice de Morisita-Horn - $\mathrm{C}_{\lambda}$ (Wolda, 1981).

As médias de abundância e riqueza dos besouros coletados foram associadas às médias dos dados de precipitação, temperatura média e umidade relativa pelo teste de correlação de Spearman - rs (Zar, 1999). Os dados climáticos mensais foram obtidos pelo IBGE (2001).

Os dados deste estudo foram comparados com outros que utilizaram o mesmo método por meio da média total de abundância e riqueza de cada armadilha em cada fitofisionomia. Foram consideradas espécies restritas aquelas com mais de dez indivíduos, que ocorreram em apenas uma das técnicas de coleta, em área aberta (campo sujo e cerrado) ou fechada (mata de galeria).

Em todos os testes estatísticos utilizados foi adotado um nível de significância de $\alpha=0,05$.

\section{Resultados e Discussão}

Foram coletados 6.879 indivíduos de 102 espécies de Scarabaeidae $s$. str. com maior proporção de besouros na armadilha de queda com isca de fezes, e as armadilhas de queda coletaram mais indivíduos e espécies no campo sujo que nas outras fitofisionomias (Tabela 1). Os dados de abundância de cada área não apresentam distribuição normal: campo sujo $\left(\mathrm{K}^{2}=117,44, \mathrm{p}<0,05\right)$, cerradosensu stricto $\left(\mathrm{K}^{2}=57,77, \mathrm{p}<0,05\right)$ e mata de galeria $\left(\mathrm{K}^{2}=79,74\right.$, $\mathrm{p}<0,05)$.

Em amostras de comunidades nos trópicos, a maioria dos indivíduos pertencem a poucas espécies enquanto, a maior parte das espécies são representadas por poucos indivíduos (Hughes, 1996). Esse padrão foi encontrado para os Scarabaeidae s. str. em vários estudos (Howden \& Nealis, 1975; Halffter et al., 1992) e corroborado para os Scarabaeidae s. str. do Cerrado neste trabalho (Figura 1).

Não houve diferença das médias de espécies $(\mathrm{H}=1,39, \mathrm{p}>0,05)$ e indivíduos $(\mathrm{H}=1,75, \mathrm{p}>0,05)$ entre as três técnicas de coleta no campo sujo (Tabela 2). Entretanto houve diferença entre os índices de diversidade: "janela" e carcaça $(\mathrm{t}=4,31, \mathrm{p}<0,05)$; "janela" e fezes $(\mathrm{t}=4,88, \mathrm{p}<0,05)$ e carcaça e fezes $(\mathrm{t}=17,11, \mathrm{p}<0,05)$; carcaça teve a menor diversidade; “janela” apresentou menos espécies e foi a armadilhacom menos espécies em comum com as demais; a maior similaridade encontrada foi entre carcaça efezes e amenorentre fezes e a "janela" (Tabela 3).

Tabela 1. Número de espécies (E) e indivíduos (I) de besouros da família Scarabaeidae e espécies restritas (ER) coletados em armadilha de interceptação de vôo do tipo "janela" e armadilha de queda com iscas de carcaça e de fezes humanas, em três fitofisionomias do Cerrado.

\begin{tabular}{|c|c|c|c|c|c|c|c|c|c|c|}
\hline \multirow{2}{*}{ Armadilha } & \multicolumn{2}{|c|}{ Campo sujo } & \multicolumn{2}{|c|}{ Cerrado } & \multicolumn{2}{|c|}{ Mata } & \multicolumn{2}{|c|}{ Total } & \multicolumn{2}{|c|}{ ER/armadilha } \\
\hline & $E$ & I & E & I & E & I & E & I & $E$ & I \\
\hline Janela & 30 & 211 & 26 & 241 & 18 & 132 & 49 & 584 & 2 & 34 \\
\hline Carcaça & 33 & 1102 & 26 & 351 & 11 & 76 & 49 & 1529 & 3 & 32 \\
\hline Fezes & 62 & 3131 & 46 & 1287 & 22 & 348 & 80 & 4766 & 7 & 192 \\
\hline Total & 80 & 4444 & 57 & 1879 & 28 & 556 & 102 & 6879 & & \\
\hline
\end{tabular}


As três técnicas, no cerrados. str., não apresentaram diferenças em suas riqueza $(\mathrm{H}=1,12, \mathrm{p}>0,05)$ eabundância $(\mathrm{H}=1,42, \mathrm{p}>0,05)$ (Tabela 2). Mas houve diferença entre os índices de diversidade de "janela" e carcaça $(\mathrm{t}=2,35$, $p<0,05)$, "janela" efezes $(t=7,60, p<0,05)$ e carcaça e fezes $(t=6,96, p<0,05)$. A "janela" tevea menor diversidade $e$ fezes apresentou menos espécies em comum com as demais (Tabela 3).

Na mata de galeria, não houve diferença entre as médias de riqueza $(H=1,20, p>0,05)$ e abundância das técnicas de coleta $(H=1,23, p>0,05)$ (Tabela 2). Entretanto, foi verificada a diferença entre os índi-

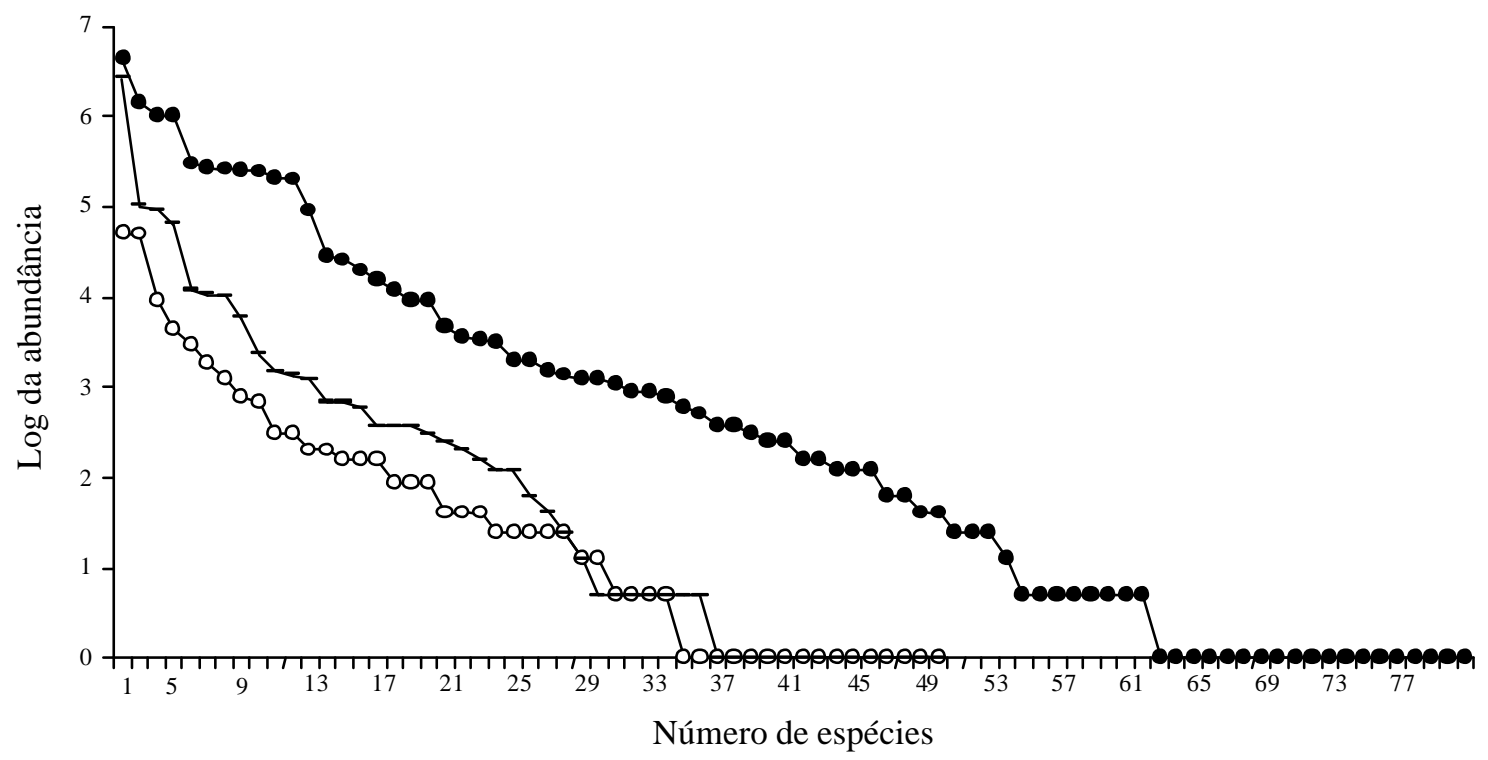

Figura 1. Curva de dominância de diversidade de espécies de besouros da família Scarabaeidae $s$. str. coletados em armadilhas de interceptação de vôo do tipo "janela" ( $($ ), e em armadilha de queda com isca de carcaça (-) e com isca de fezes ( ), em três fitofisionomias do Cerrado de Brasília.

Tabela 2. Número de espécies (riqueza) e de indivíduos (abundância) por armadilha e por dia de coleta de besouros da família Scarabaeidae coletados em armadilhas de interceptação de vôo do tipo "janela" (J) e armadilhas de queda com iscas de carcaça $(\mathrm{C})$ e de fezes humanas $(\mathrm{F})$ em três fitofisionomias do Cerrado.

\begin{tabular}{|c|c|c|c|c|c|c|c|c|c|}
\hline \multirow[t]{2}{*}{ Mês/ano } & \multicolumn{3}{|c|}{ Campo sujo } & \multicolumn{3}{|c|}{ Cerrado s. str. } & \multicolumn{3}{|c|}{ Mata de galeria } \\
\hline & $\mathrm{J}$ & $\mathrm{C}$ & $\mathrm{F}$ & $\mathrm{J}$ & $\mathrm{C}$ & $\mathrm{F}$ & $\mathrm{J}$ & $\mathrm{C}$ & $\mathrm{F}$ \\
\hline & & & & & Espécies & & & & \\
\hline 10/1999 & 8,5 & 3,6 & 8,2 & 10,5 & 3,4 & 6,8 & 6,0 & 1,8 & 2,6 \\
\hline $11 / 1999$ & 9,5 & 4,0 & 9,8 & 5,0 & 1,2 & 5,6 & 4,0 & 1,8 & 2,8 \\
\hline $12 / 1999$ & 3,0 & 3,2 & 7,2 & 5,0 & 4,0 & 4,6 & 3,0 & 0,4 & 1,2 \\
\hline $01 / 2000$ & 6,0 & 4,4 & 6,2 & 3,5 & 2,6 & 3,8 & 3,0 & 0,6 & 1,2 \\
\hline $05 / 2000$ & 0,5 & 0,2 & 0,6 & - & 0,2 & 1,4 & - & - & 0,6 \\
\hline $06 / 2000$ & 0,5 & - & 0,6 & - & - & 0,2 & - & - & - \\
\hline $07 / 2000$ & - & - & - & - & - & - & - & - & 0,2 \\
\hline $08 / 2000$ & - & - & - & - & - & - & - & - & - \\
\hline Total & 0,19 & 0,08 & 0,16 & 0,16 & 0,07 & 0,12 & 0,11 & 0,03 & 0,06 \\
\hline 10/199 & 245 & 810 & 1346 & 780 & Indivíduo & 060 & 425 & 054 & 264 \\
\hline $11 / 1999$ & $\begin{array}{l}2,4 \mathrm{~S} \\
3,65\end{array}$ & $\begin{array}{l}8,10 \\
5,06\end{array}$ & $\begin{array}{l}15,40 \\
19,80\end{array}$ & $\begin{array}{l}1,80 \\
1,40\end{array}$ & $\begin{array}{l}3,54 \\
0,58\end{array}$ & $\begin{array}{l}9,60 \\
7,32\end{array}$ & $\begin{array}{l}4,25 \\
1,05\end{array}$ & $\begin{array}{l}0,34 \\
0,70\end{array}$ & $\begin{array}{l}2,64 \\
3,21\end{array}$ \\
\hline $12 / 1999$ & 1,50 & 4,10 & 12,24 & 1,90 & 1,84 & 4,08 & 0,90 & 0,20 & 0,38 \\
\hline $01 / 2000$ & 2,85 & 4,76 & 16,84 & 0,95 & 1,04 & 4,52 & 0,40 & 0,08 & 0,56 \\
\hline $05 / 2000$ & 0,05 & 0,02 & 0,20 & - & 0,02 & 0,20 & - & - & 0,24 \\
\hline $06 / 2000$ & 0,05 & - & 0,08 & - & - & 0,02 & - & - & - \\
\hline $07 / 2000$ & - & - & - & - & - & - & - & - & 0,02 \\
\hline $08 / 2000$ & - & - & - & - & - & - & - & - & - \\
\hline Total & 1,31 & 2.75 & 7,82 & 1,50 & 0,87 & 3,21 & 0,82 & 0,19 & 0,87 \\
\hline
\end{tabular}


ces de diversidade de "janela" e fezes $(\mathrm{t}=4,26, \mathrm{p}<0,05)$ e carcaça e fezes $(t=3,44, p<0,05)$, com exceção de "janela" e carcaça $(t=0,68, p>0,05)$. Fezes apresentou a menor diversidade e tem menos espécies em comum com as demais (Tabela 3).

Houve diferença entre técnicas e fitofisionomias quanto à abundância $\left(\chi^{2}=314,05 ; \mathrm{p}<0,05\right)$, o que não ocorreu com a riqueza $\left(\chi^{2}=2,439 ; p>0,05\right)$ (Tabela 1$)$. As maiores similaridades foram entre carcaça e "janela" no cerrado e na mata, enquanto a menor foi entre "janela" e fezes no campo sujo (Tabela 3).

A eficiência das armadilhas pode variar segundo a fitofisionomia. Para os escarabeídeos, a cobertura vegetal afeta as condições microclimáticas, principalmente nos trópicos (Halffter, 1991; Halffter et al., 1992). No Cerrado, a maior diversidade ocorreu no campo sujo que é a área mais aberta de todas, e isto corrobora os resultados encontrados em estudos realizados em floresta e savana africana. Entretanto, a explicação para a maior diversidade na África (maior disponibilidade de alimento proporcionada por rebanhos de grandes mamíferos) não se aplica ao Cerrado, que possui uma fauna de mamíferos de pequeno porte (Marinho Filho et al., 2002).

Existem dúvidas se as coletas com armadilhas se aproximam da realidade e, no caso dos escarabeídeos,

Tabela 3. Índices de diversidade de Shannon (H'), de equitabilidade (J') e de similaridade de Morisita $\left(\mathbf{C}_{\lambda}\right)$ obtidos com os dados de coleta de besouros da família Scarabaeidae s. str. em armadilhas de interceptação de vôo do tipo "janela" e armadilhas de queda com iscas de carcaça e de fezes humanas, em três fitofisionomias do Cerrado.

\begin{tabular}{|c|c|c|c|c|c|}
\hline \multirow[t]{2}{*}{ Armadilha } & \multirow{2}{*}{$\begin{array}{c}\text { Diversidade } \\
\left(\mathrm{H}^{\prime}\right)\end{array}$} & \multirow{2}{*}{$\begin{array}{c}\text { Equitabilidade } \\
\left(\mathrm{J}^{\prime}\right)\end{array}$} & \multicolumn{3}{|c|}{ Similaridade $(\mathrm{C} \lambda)$} \\
\hline & & & Janela & Carcaca & Fezes \\
\hline & \multicolumn{5}{|c|}{ Campo sujo } \\
\hline Janela & 1,079 & 0,731 & 1,000 & & \\
\hline Carcaça & 0,899 & 0,592 & 0,457 & 1,000 & \\
\hline \multirow[t]{2}{*}{ Fezes } & 1,266 & 0,706 & 0,247 & 0,792 & 1,000 \\
\hline & \multicolumn{5}{|c|}{ Cerrado sensu stricto } \\
\hline Janela & 0,961 & 0,679 & 1,000 & & \\
\hline Carcaça & 1,064 & 0,752 & 0,894 & 1,000 & \\
\hline Eezes & 1.252 & 0.753 & 0.583 & 0.703 & .1 .000 \\
\hline & \multicolumn{5}{|c|}{ Mata de galeria } \\
\hline Janela & 0,882 & 0,623 & 1,000 & & \\
\hline Carcaça & 0,839 & 0,805 & 0,831 & 1,000 & \\
\hline Fezes & 0.640 & 0.476 & 0.670 & 0.515 & 1,000 \\
\hline
\end{tabular}

há discussões sobre se existe maior diversidade em áreas abertas ou se as armadilhas são mais eficientes. A presença de 31 espécies restritas com 4.865 indivíduos na área aberta (campo sujo e cerrado) e de 4 espécie restritas com 397 indivíduos na área fechada (mata de galeria), sugere que as áreas abertas têm mais espécies do que as áreas fechadas (Tabela 1). Essa diferença na diversidade de besouros refletiu-se nas coletas das três armadilhas nas três fitofisionomias. Uma das possíveis explicações para as diferenças de diversidade entre áreas abertas e fechadas do Cerrado pode ser o tamanho pequeno e o is olamento das matas de galeria, já que a maioria dos habitats desse bioma é de áreas abertas (Eiten, 1972). Comparando-se dados de Scarabaeidae s. str. entre florestas da Colômbia, Borneo e Uganda encontrou-se menor diversidade nas florestas de Uganda. Este resultado foi explicado pela pequena área das florestas e sua ocorrência em "manchas", o que promove isolamento (Hanski, 1983; Nummelin \& Hanski, 1989).

Pesquisas feitas em outros ecossistemas com técnicas similares às deste trabalho mostram maiores médias/armadilha/dia de besouros em mata primária (0,24 espécies e 2,41 indivíduos em carcaça e 0,42 espécies e 10,78 indivíduos em fezes) (Lopes et al., 1994); seguida por restinga (0,08 espécies e 4,04 indivíduos em carcaça e 0,11 espécies e 18,73 indivíduos em fezes) (Louzada et al., 1996); e por floresta secundária $(0,001$ espécie e 0,13 indivíduo em carcaça e 0,01 espécie e 0,33 indivíduo em fezes) (Louzada $\&$ Lopes, 1997). As maiores médias de riqueza e abundância em armadilhas de queda foram encontradas em fezes e os maiores índices de diversidade em fezes no campo sujo e cerrado s. str. (Tabelas 2 e 3 ). Os trabalhos citados mostraram que a armadilha de queda com isca de fezes humanas foi a maisadequada ao estudo dos escarabeídeos, independentemente do ecossistema estudado, pois em todos houve maior riqueza de espécies nesse tipo de armadilha.

No Cerrado, a maior proporção de espécies e indivíduos foi obtida nas coletas da armadilha com fezes (77,6\%) não só com grande número de espécies res- 
tritas mas, também, com muitas espécies em comum com carcaça (Tabelas 1 e 3). Esse dado corrobora o resultado de outros estudos nos quais essa isca também foi a mais atrativa (Howden \& Nealis, 1975; Lopes et al., 1994; Louzada et al., 1996; Louzada \& Lopes, 1997).

Houve associação positiva entre as médias de espécies e de indivíduos com a precipitação e com a umidade relativa, com exceção de carcaça em mata de galeria com umidade relativa. Entretanto, não houve correlação entre a diversidade e temperatura média (Tabelas 4 e 5). O resultado deste trabalho, de maior frequência de espécies e indivíduos de Scarabaeidae s. str. no Cerrado em 1999/2000 na estação chuvosa, corrobora resultados encontrados em outros locais do Brasil (Lopes et al., 1994; Flechtmann et al., 1995; Louzada \& Lopes, 1997) e do mundo (Halffter \& Matthews, 1966; Hanski \& Cambefort, 1991).

Tabela 4. Médias ( \pm desvio-padrão) dos valores de precipitação, temperatura e umidade relativa referentes a dez dias de coleta a cada mês.

\begin{tabular}{cccc}
\hline Meses/ano & $\begin{array}{c}\text { Precipitação } \\
(\mathrm{mm})\end{array}$ & $\begin{array}{c}\text { Temperatura } \\
\left({ }^{\circ} \mathrm{C}\right)\end{array}$ & $\begin{array}{c}\text { Umidade relativa } \\
(\%)\end{array}$ \\
\hline $10 / 1999$ & $11,19(3,72)$ & $21,79(0,48)$ & $78,07(2,91)$ \\
$11 / 1999$ & $8,29(3,13)$ & $22,14(0,37)$ & $79,14(3,03)$ \\
$12 / 1999$ & $4,61(2,57)$ & $22,89(0,37)$ & $77,27(2,46)$ \\
$01 / 2000$ & $3,10(1,21)$ & $22,51(0,44)$ & $83,10(2,29)$ \\
$05 / 2000$ & 0,00 & $21,17(0,39)$ & $59,90(1,74)$ \\
$06 / 2000$ & 0,00 & $20,88(0,23)$ & $55,80(2,86)$ \\
$07 / 2000$ & 0,00 & $21,64(0,34)$ & $48,50(2,29)$ \\
$08 / 2000$ & $1,13(0,93)$ & $23,24(1,09)$ & $49,40(6,68)$ \\
\hline
\end{tabular}

Em levantamentos das principais espécies de Scarabaeidae s. str., a armadilha de queda com fezes é a mais indicada para se efetuar coletas representativas da comunidade local. Além disso, apresenta facilidade na obtenção da isca e possui logística mais fácil do que a de "janela".

Entretanto, para se obter dados mais precisos sobre a composição de espécies da comunidade de escarabeídeos, é melhor utilizar uma combinação de métodos como coletas manuais e armadilhas com outras iscas, já que nem todas as espécies respondem a uma determinada armadilha e muitos escarabeídeos considerados raros podem ser foréticos de alguns animais ou mimercófilos (Halffter \& Matthews, 1966; Favila \& Halffter, 1997).

Este trabalho é o primeiro levantamento da fauna de Scarabaeidae s. str. na região do DF, contribuindo para a construção do conhecimento básico dessa fauna nos Cerrados. Alguns aspectos da fauna de Scarabaeidae s. str. podem ser considerados em pesquisas futuras, como o levantamento da fauna em cada um dos diversos tipos fitofisionômicos do Cerrado, compilação de uma lista de espécies que ocorram nesse bioma e elaboração de uma chave de identificação para as espécies do Distrito Federal.

Tabela 5. Correlação entre as médias de espécies (E) e de indivíduos (I) de besouros da família Scarabaeidae s. str. coletados em armadilhas de interceptação de vôo do tipo "janela" e armadilhas de queda com iscas de carcaça e de fezes humanas e variáveis climáticas em três fitofisionomias do Cerrado ${ }^{(1)}$.

\begin{tabular}{|c|c|c|c|c|c|c|}
\hline \multirow[t]{2}{*}{ Armadilha } & \multicolumn{2}{|c|}{ Precipitacão $(\mathrm{mm})$} & \multicolumn{2}{|c|}{ Temperatura média $\left({ }^{\circ} \mathrm{C}\right)$} & \multicolumn{2}{|c|}{ Umidade relativa $(\%)$} \\
\hline & $\mathrm{E}$ & $\mathrm{I}$ & $\mathrm{E}$ & $\mathrm{I}$ & $\mathrm{E}$ & I \\
\hline $\begin{array}{l}\text { Janela } \\
\text { Carcaça } \\
\text { Eezes }\end{array}$ & $\begin{array}{l}0,9167^{* *} \\
0,8095^{*} \\
0.9405^{* *}\end{array}$ & $\begin{array}{l}0,8452^{\text {** }} \\
0,9405^{\text {** }} \\
0.7857^{*}\end{array}$ & $\begin{array}{l}{ }_{0,3452}{ }^{\mathrm{Ca}} \\
0,4524^{\mathrm{ns}} \\
0,3690^{\mathrm{ns}}\end{array}$ & $\begin{array}{l}0,4286^{\mathrm{ns}} \\
0,2857^{\mathrm{ns}} \\
0.3571^{\mathrm{ns}}\end{array}$ & $\begin{array}{l}0,8690^{\text {** }} \\
0,9286^{* *} \\
0.7976^{*}\end{array}$ & $\begin{array}{l}0,9167^{* * *} \\
0,8452^{* * *} \\
0.9524^{* *}\end{array}$ \\
\hline $\begin{array}{l}\text { Janela } \\
\text { Carcaça } \\
\text { Fezes } \\
\end{array}$ & $\begin{array}{l}0,9583^{\text {*** }} \\
0,8452^{\text {** }} \\
0,9048^{\text {** }}\end{array}$ & $\begin{array}{l}0,9405^{\text {*** }} \\
0,8929^{\text {** }} \\
0,8810^{\text {** }}\end{array}$ & $\begin{array}{r}\text { Cer } \\
0,3750^{\text {ns }} \\
0,4881^{\text {ns }} \\
0,2381^{\text {ns }} \\
\end{array}$ & $\begin{array}{l}0,2857^{\mathrm{ns}} \\
0,2857^{\mathrm{ns}} \\
0,2143^{\mathrm{ns}}\end{array}$ & $\begin{array}{l}0,7560^{*} \\
0,7262^{*} \\
0,8095^{*} \\
\end{array}$ & $\begin{array}{l}0,7262^{*} \\
0,7500^{* *} \\
0,8810^{* *}\end{array}$ \\
\hline $\begin{array}{l}\text { Janela } \\
\text { Carcaça } \\
\text { Fezes }\end{array}$ & $\begin{array}{l}0,9583^{\text {** }} \\
0,8095^{* *} \\
0.9524^{\text {** }}\end{array}$ & $\begin{array}{l}0,9643^{* *} \\
0,8810^{* *} \\
0.8571^{*}\end{array}$ & $\begin{array}{l}\text { Mat } \\
0,3393^{\mathrm{ns}} \\
0,2143^{\mathrm{ns}} \\
0.3571^{\mathrm{ns}}\end{array}$ & $\begin{array}{l}\text { ia } \\
0,2857^{\mathrm{ns}} \\
0,3095^{\mathrm{ns}} \\
0.2857^{\mathrm{ns}}\end{array}$ & $\begin{array}{l}0,8155^{*} \\
0,5952^{\mathrm{ns}} \\
0.8333^{*}\end{array}$ & $\begin{array}{l}0,7738^{*} \\
0,5952^{\text {ns }} \\
0.9048^{*}\end{array}$ \\
\hline
\end{tabular}




\section{Conclusões}

1. A armadilha de queda com isca de fezes humanas é a mais eficiente para coletar as principais espécies de besouros coprófagos da família Scarabaeidae s. str.

2. As maiores ocorrências de espécies e indivíduos de Scarabaeidae $s$. str. são registradas na estação das chuvas, nas três fitofisionomias estudadas e nos três tipos de armadilha utilizados.

3. O campo sujo é a fitofisionomia do Cerrado do Distrito Federal que apresenta maior número de espécies e indivíduos de Scarabaeidae s. str.

\section{Agradecimentos}

A Andrei Polejack, pela leitura crítica dos manuscritos e pelas sugestões pertinentes; aos técnicos do Departamento de Zoologia da UnB, Maria do Perpétuo Socorro Bandeira e Santos Balbino, pela ajuda na coleta e triagem dos insetos; ao corpo administrativo da Reserva Ecológica do IBGE e ao CNPq, pelo apoio financeiro à primeira autora.

\section{Referências}

CAMPOS, W. G.; PEREIRA, D. B. S.; SCHOEREDER, J. H. Comparison of the efficiency of flight-interception trap models for sampling Hymenoptera and other insects. Anais da Sociedade Entomológica do Brasil, Piracicaba, v. 29, n. 3 , p. $381-389,2000$

EITEN, G. The cerrado vegetation of Brazil. Botanical Review, New York, v. 38, p. 201-341, 1972.

FAVILA, M. E.; HALFFTER, G. The use of indicator groups for measuring biodiversity as related to community structure and function. Acta Zoológica Mexicana, México, v. 72, p. 1-25, 1997

FLECHTMANN, C. A. H.; RODRIGUES, S. R.; COUTO, H. T. Z. Controle biológico da mosca-dos-chifres (Haematobia irritans irritans) em Selvíria, Mato Grosso do Sul - 4: comparação entre métodos de coleta de besouros coprófagos (Scarabaeidae). Revista Brasileira de Entomologia, São Paulo, v. 39, n. 2, p. 259-276, 1995.

HALFFTER, G. Historical and ecological factors determining the geographical distribution of beetles (Coleoptera: Scarabaeidae: Scarabaeinae). Folia Entomológica Mexicana, México, v. 82, p. 195-238, 1991.
HALFFTER, G.; FAVILA, M. E.; HALFFTER, V. A comparative study of the structure of the scarab guild in Mexican tropical rain forests and derived ecosystems. Folia Entomológica Mexicana, México, v. 84, p. 131-156, 1992.

HALFFTER, G.; MATTHEWS, E. G. The natural history of dung beetles of the subfamily Scarabaeinae (Coleoptera: Scarabaeidae). Folia Entomológica Mexicana, México, v. 12 , n. 14 , p. $1-312,1966$.

HANSKI, I. Distributional ecology and abundance of dung and carrion-feeding beetles (Scarabaeidae) in tropical rain forests in Sarawak, Borneo. Acta Zoologica Fennica, Helsinki, v. 167, p. 1-45, 1983.

HANSKI, I.; CAMBEFORT, Y. (Ed.). Dung beetle ecology. Princeton: Princeton University Press, 1991. $481 \mathrm{p}$.

HERTEL, F.; COLLI, G. R. The use of leaf cutter ants, Atta laevigata (Smith) (Hymenoptera: Formicidae), as a substrate for oviposition by dung beetle Canthon virens Mannerheim (Coleoptera: Scarabaeidae) in Central Brazil. Coleopterists Bulletin, Natchez, v. 52, n. 2, p. 105-108, 1998

HOWDEN, H. F.; NEALIS, V. G. Effects of clearing in a tropical rain forests on the composition of the coprophagous scarab beetle fauna (Coleoptera). Biotropica, Lawrence, v. 7, n. 2, p. 77-83, 1975.

HUGHES, R. G. Theories and models of species abundances. American Naturalist, Chicago, v. 128, p. 879-899, 1996.

IBGE. Reserva Ecológica. Estação Meteorológica. Base de dados: dados climáticos. Disponível em: <http:// www.recor.org.br/Estacao/consulta.asp >. Acesso em: 11 fev. 2001.

LOPES, J.; CONCHON, I.; YUZAWA, S. K.; KURLEIN, R. R. C. Entomofauna do Parque Estadual Mata dos Godoy - II: Scarabaeidae (Coleoptera) coletados em armadilhas de solo. Semina Ciências Biológicas e Saúde, Londrina, v. 15, n. 2, p. 121-127, 1994.

LOUZADA, J. N. C.; LOPES, F. S. A comunidade de Scarabaeidae copronecrófagos (Coleoptera) de um fragmento de Mata Atlântica. Revista Brasileira de Entomologia, São Paulo, v. 41, n. 1, p. 117-121, 1997.

LOUZADA, J. N. C.; SHIFFLER, G; MELLO, F. Z. V. de. Efeitos do fogo sobre a composição e estrutura da comunidade de Scarabaeidae (Insecta: Coleoptera) da restinga da ilha de Guriri, Norte do Espírito Santo. In: SIMPÓSIO IMPACTOS DE QUEIMADAS SOBRE OS ECOSSISTEMAS E MUDANÇAS GLOBAIS, 3.; 
CONGRESSO DE ECOLOGIA DO BRASIL, 1996, Brasília. Anais... Brasília: UnB, 1996. p. 161-169.

MARINHO FILHO, J. S.; RODRIGUES, F. H. G.; JUAREZ, K. M. The cerrado mammals: diversity, ecology and natural history. In: OLIVEIRA, P. S.; MARQUIS, R. J. (Ed.). The Cerrado of Brazil: ecology and natural history of a neotropical savanna. New York: Columbia University Press, 2002. p. 266-284.

MITTERMEIER, R. A.; MYERS, N.; MITTERMEIER, C. G. (Ed.). Hotspots: earth's biologically richest and most endangered terrestrial ecoregions. México: CEMEX/ Conservation International, 2000. 431 p.

MONTREUIL, O. Phylogenetic analysis and paraphyly of Coprini and Dichotomiini (Coleoptera: Scarabaeidae): biogeographic scenario. Annales de la Société Entomologique de France, Paris, v. 34, n. 2, p. 135-148, 1998.

NUMMELIN, M.; HANSKI, I. Dung beetles of the Kibale forest, Uganda: comparison between virgin and managed forests. Tropical Ecology, Cambridge, Inglaterra, v. 5, p. 349-352, 1989.

PINHEIRO, F.; DINIZ, I. R.; COELHO, D.; BANDEIRA, M. P. S. Seasonal pattern of insect abundance in the Brazilian cerrado. AustralEcology, Carlton, v. 27, p. 132136, 2002.

SHEPERD, V. E.; CHAPMAN, C. A. Dung beetles as secondary seed dispersers: impact on seed predation and germination. Tropical Ecology, Cambridge, Inglaterra, v. 14, p. $199-215,1998$.

WATERHOUSE, D. F. The biological control of dung. Scientific American, New York, v. 230, n. 4, p. 100-109, 1974.

WOLDA, H. Similarity indices, sample size and diversity. Oecologia, New York, v. 50, p. 296-302, 1981.

ZAR J. H. Biostatistical analysis. Upper Saddle River: Prentice-Hall, 1999. 662 p.

ZUNINO, M. Las relaciones taxonómicas de los Phanaeina y sus implicaciones biogeográficas. Folia Entomológica Mexicana, México, v. 64, p. 101-115, 1985. 